\title{
LA MEDERSA * DES MORISCOS ANDALOUS À TUNIS **
}

Per

ABDEL-HAKIM EL GAFSI

Les travaux relatifs à cette institution musulmane, à son origine, à sa portée politique et religieuse ainsi qu' à son évolution sont connus (1).

Née à Nisapour vers l'année 1009 (2), elle apparaît à Baghdad en 1064 (3), à Damas en 1097, à Alep vers 1120 (4), à Alexandrie en 1151, au Caire en 1170, à Tunis vers 1249, à Fez en 1280 et à Grenade en 1349. Par conséquent, elle a mis plus de 340 ans pour passer de l'Orient à l'Andalousie.

(") A l'origine lâge medersa etait une institution purement scolaire; elle correspondait un peu pres au collège du moyen occidental.

En Tunisie, ce terme fut employé au XIX e siècle pour designer un collège comme ce fut le cas de la medersa aș șādikiyya crée en 1874 (Abdesselem, A, les historiens tunisiens, París, Klincksieck, 1973; 60, 138, 139 ). Liman, R siyāsat Hamūda bāŠa, 1782-1814, Tunis, Faculté deslettres, 1980, 488.

Ou une academie militaire comme ce fut le cas de madrasat bārdū al Ḥarbiyya (Abd al Mula, Madrasat Bardu, Tunis, Maison arabe du livre, 1977, 162. P. BNT, MS 4271.

Ou internat; c'est ainsi que la medersa des andalous se transforma en lieu d'habitation pour les etudiants algeriens inscrits à La Zitouna (Ben Mami, B, Madāris Tūnis fi al 'ahdayn al Hafsĩ wa at turkī, Tunis, 1983, 'Faculté desletres, p. 285.

Il faut noter aussi que les medersas ont servi de lieu de refuge pour les moriscos andalous refugiés en Tunisie apres l'edit d'expulsión de Philippe III en 1609 (Zbips, SM, Gafsi, A, Boughanmi, M. Epalza, M. Etudes sur les morisques andalous, Tunis, Inaa, 1983, 123.

Ce terme peut designer aussi un courant litteraire ou théologique (lbn aš šmmăc, mort en 1435. 'al 'adilla, Tunis, Maison arabe du livre, 1984, 7.

(*) Appelée al madrasa al andalusiyya, madrasat sīdī 'al cağmĩ à cause de la tombe de ce saint qui se trouvait en face; madrasat al Fatḥ comme l'indique l'inscription, madrasat aš Sayn Saacbān 'al andalusĩ qui fut son premier professeur.

(1) Brunschvig, R. quelques remarques historiques sur les medersas en Tunisie, RT 6, 1*931, 261-285.

(2) İdem

(3) 'Al Mašadānī, M. J H, Nakšabandī, U N, al Mustanșiriyya, Baghdad, 1986, 232 p.

(4) Juha, F, al madaris al aṭariyya fī Ḥalab, Awraq, 5-6, 1982-1983, 65-78. 
En tant qu'institution d'enseignement supérieur, elle ne pouvait être qu'un instruemnt idéologique et politique aux mains du pouvoir dominant. C'est ce que nous allons essayer d'expliquer trés brievèment au cours de l'étude des medersas en Tunisie.

\section{Les medersas en Tunisie}

L'implantation de cette institution en Tunisie remonte au XIII e siècle; en effet au cours de ce siècle le pouvoir hafside (1227-1530) fonda à Tunis trois medersas: la šmmāicyya en 1249 At Tawfīkiyya en 1260 et la Macridiyya en 1282 (5) dans le but de répandre le malékisme (coran et sunna); avec l'arrivée des ottomans en 1574, elle devint un instrument de la propagation du hanéfisme. En effet, c'est à partir de la medersa que le pouvoir pouvait recruter ses auxiliaires indispensables à sa marche à savoir les fonctionnaires religieux (imams..) et civils (cadis, muftis;...) Par conséquent, elle fut un courroi de promotion sociale et un instrument de contrôle de la population.

La contenu de cet enseignement «ll semble bien qu'a la fin du XVI et au début du XVII e siècles, I'enseignement se bornait à la lecture pure et simple, et dans les meilleurs cas à une paraphrase des ouvrages" (6) a beaucoup encouragé cette orientation.

Payés sur les revenus provenant des wakfs (eu habous) ou sur les produits des impôts, les enseignants ou mudarris dispensaient un enseignement quotidien aux etudiants qui recevaient de leur coté des pensions alimentaires et des dons (7).

Ces wakfs ou biens inalienables servaient aussi à l'entretien des medersas. Cet entretien (gestion materielle et surveillance des etudiants) etait confié à un cheikh (8).

S'agit-il du wakīl ou gérant rapporté par un document datant de $1186 / 1772$ et déposé dans les Archives Générales du Gouvernement Tunisien (A.G.G.T.) (9).

(5) Bruschvig, $R$ quelques. 264

(6) Abdesselem, A OP CIT, 24. Ar rașșaca (mort en 1488) Fahrast, Tunis, Afrika, 1967, 132. II faut remarquer que les autres institutions d'endeigenement comme le kuttab ou ecole coranique (Epalza, M. PETIT, Petit, R. Etudes sur les moriscos andalous eu Tunisie, Madrid, Instituto Hispano Arabe de Cuitura, 1973 105. La zawiyya ou mausalée (Dunant, H. La regence de Tunis, Tunis, STD, 1975, 138) et les autres (Ben Zid, macāris at taciìm al 'islāmi bi tūnis. In esqisse d'un bilan de la production scientifique. Tunis, Ministere de la Recherche Scientifique, 1972, 22 que je n'ai pas pu le consulter) ne diferent pas des medersas sur le plan du contenu.

(7) Abdesselem, A, OP CIT, 400. Cherif, MH, L'histoire économique et sociale de la Tunisieau XVIli e siecle. In "Les arabes par leurs archives", París, CNRS, 1976, 111. Ricard, P, pour comprende l'art musulman dansi Áfrique du Nord et en Espagne; París, Hachette, 1924, 208. Brunchvig, R quelques remarques, 278

(8) Bruschvig, $R$, quelques remarques; 278

(9) AG. G. T registre 2305, P5. Wakīl de la medersa al slimāniyya. 
Naturellement, ce statut fut modifié au moment du Protectorat français en Tunisie en 1881. En effet, les decrets du 20 fevrier 1889 et celui du 25 septembre 1933 ainsi que l'arrété ministériel du 17 novembre de la même année modifient ce statut en confiant la direction de la medersa à un cheikh directeur faisant partie d'un conseil d'administration.

Voilà donc trés brièvement un aperçu sur les medersas en Tunisie et voyons maintenant la medersa des moriscos andalous.

\section{La medersa al andalousiyya (Fig. 1)}

Cette medersa se trouve en plein quartier juif ou hara (10) de Tunis et pius précisement dans la rue Ar Rakkān, par la rue de la verrerie (11); donc dans un quartier comercial mais aussi d'implantation morisco-andalouse (12).

\section{Date de fondation, fondateurs (Fig. 2)}

Sur le mur du patio opposé à celui du mihrāb est cellée une plaque de marbre comptant huit lignes de cursive en relief de style andalous, portant aprés la basmalah, la tašliyan, le nom de la medersa (13) la liste des fondateurs et la date en toutes lettres (14).

Fondée par 'Abū'al Hasan 'Alī Ibn' 'Abd Allāh Muḥammad An Nawīi, connu sous le nom d'lbn As Sarrāg (15), Muhammad B Maḥfud et Muhammad B 'Abd Al Karīm. Donc, il s'agit de représentansts importants de la colonie de moriscos andalous refugiés en Tunisie aprés l'edit d'expulsion de Philippe III, en 1609; puisque ils appartenaient à l'associat ion des nobles (Surafā 1, descendants du prophète (16).

(10) Sebag, P, I'evolution d'un gnetto nord africain: la hara de Tunis, París, P.U.F. 1969, 32. Le registre 21816 (BNT) nous renseigne sur quelques habitants juifs de ce quartier.

(11) Zbiss, S M, Les monuments de Tunis, Tunis, STD, 1971, 55.

Il ne s'agit pas de l'agrandissement de la mosquée Subhan 'allāl comme l'affirme J D latham, mais d'une création surtout que cette mosquée se trouve actuellement entre la rue bad swika et avenue ali Belhouane alors que la medersa se trouve un peu loin. CT, 19-20, 1957, 228. Epalza, M, Petit; r, OP cit, 45. et Zbiss, Gafsi, Boughanmi, Epalza, op cit. p, 161

Cette medersa se trouve non loin de la Turba de sīdi younis mort en 1106 qui fut la maitre de sidi Muhriz protecteur de Tunis. Ar rașșca, Op. Cit. 173. Ibn abī diāt, Ithāâ, Tunis, STD, 1976, Vol. 2,35.

(12) Gafsi, A, note sur l'archeologie: source de I'histoire morisco-andalouse, In actes du II Symposieum International de C.I.EM, Tunis, 1984, 303.

(13) Cette insciption fut reproduite par Ben el Khodja, Tāriḥ, Beyrouth, dar al gharb al islami, 1985, 297 et Ben Mami, B, op. cit., 363 qui ont omis de mettre les deux derniera mots de la 6 e ligne (Wa mina aț țâgrī). Faut il lire ce mot at țâgrī qui veut dire ftontiere (Epalza, M. Petit, R, op. cit., p. 279).

Elle est nommée madrasat al fath. Ce mot, d'aprés coran CX, 1 (I'orsque l'assistance de Dieu et la victoire nous arrivent (París, Flammarion, 1970, traduction de Kasimirski) indique la victoire et par conséquent l'espoir, de mener une vistoire sur les «infideles» qui permettra de retourner en Espagne. Ce mot fut adoptée aussi par une des composantes de l'organisation de la liberation de la palestine (fath) pour exprimer, croyons nous, le meme espoir.

(14) Zbiss, monuments de Tunis, 55.

(15) Cette famille donna un grand historien du XVII e siècle (Abdesselem; A, op. cit., 221. Epalza, M, Petit, R, op. cit, 268.

(16) Zbiss, Gafsi, Boughanmi, Epalza, op. cit., 25. 161. 


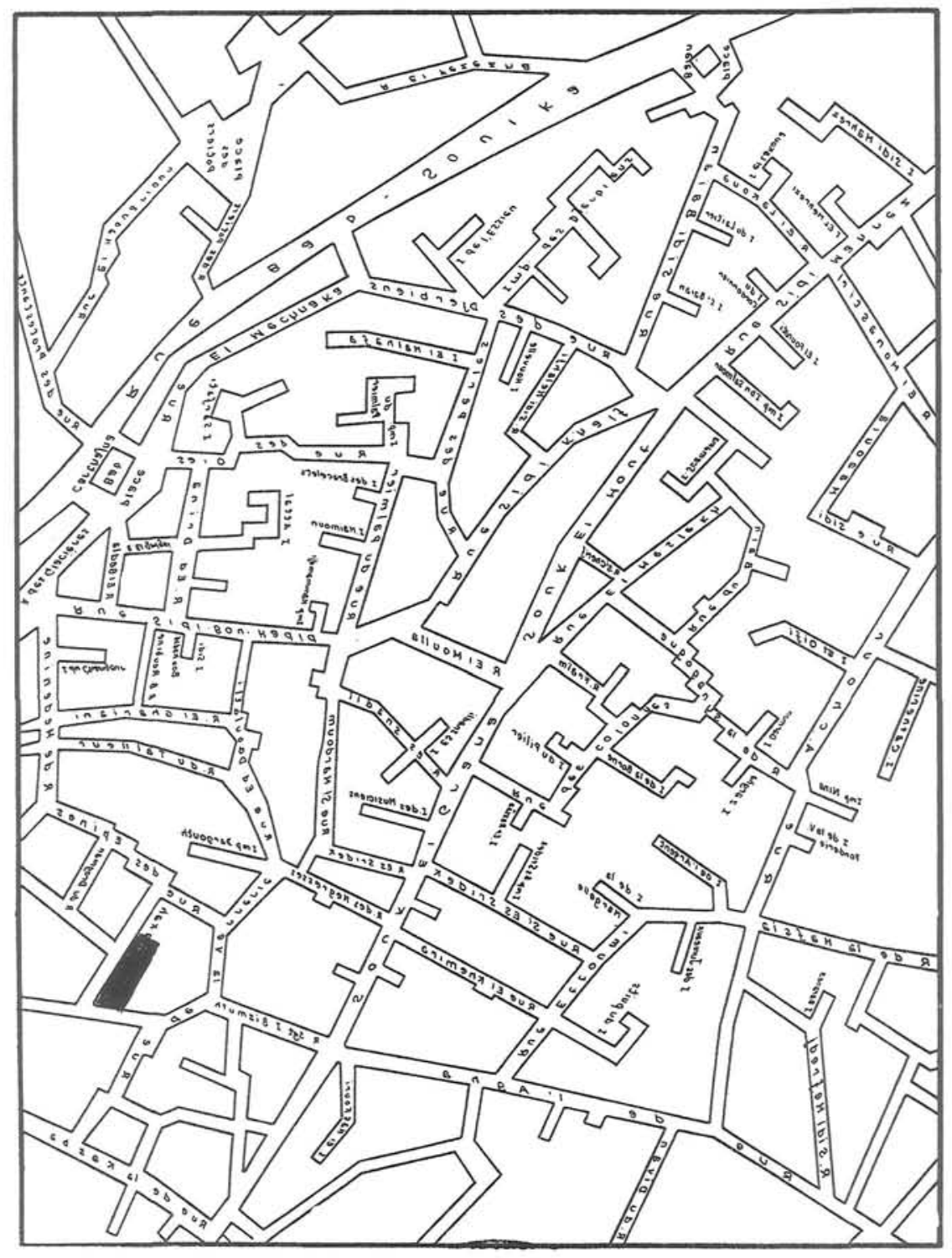

Fig. 1. Situation de la Médersa des Andalous dans le quartier de la Hara, avant les efforts d'assainissement, selon P. Sebag. 


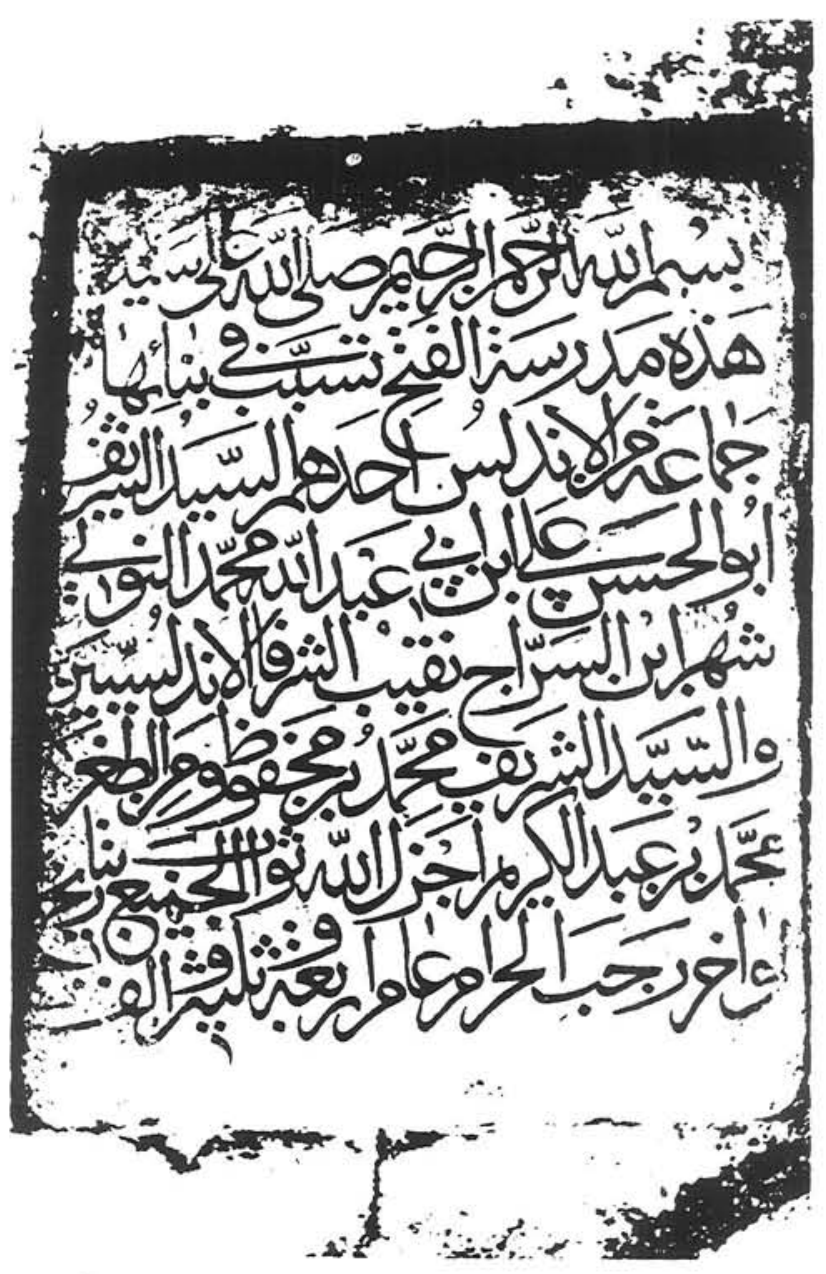

Fig. 2. Inscription à la Médersa des Andalous. 
Notons aussi que le premier fut le syndic (naheïb) de cette association. Cette fonction importante fut à plusieurs reprises le privilg̀e de familles d'origine morisco andalous e; parmi elles, nous pouvons citer Tarwal (Teruel) (17) et Bin ${ }^{\mathrm{C}} \mathrm{Ašu} r(18)$.

Ce nakīb faisait partie, à l'epoque ottomane du conseil de justice (Mağlis' al Kadā) compose du Bāša, du cadi et du mufti (la jurisconsulte) (19). A kairouan ce syndic rece vait des traitements reguliers relevés sur le produit des impôts de la ville (20). Le dernier cité dans l'inscription est un ecrivain bien connu (21).

Cette medersa fut fondée (achevée) dans les derniers jours du mois de Rağab 1034 / mai 1625; c'est-à-dire sous le règne de Youssef Dey (16101637) qui fut parmi les (22) fervents protecteurs de cette colonie.

\section{Enseignants:}

Le premier professeur de la medersa fut Ša ${ }^{\complement} a$ bān' al 'andalusí (23) versé dans la science du kalām (la rhétorique) (24); il fut remplacé à sa mort par un autre andalous, 'Abū ar Rabī Sulaymān al 'Andalusī, le grand grammairien (nahwi) (25).

Aprés eux, nous n'avons pas pu relever d'autres andalous, d'aprés les documents à notre disposition (26).

Mais il faut remarquer que les andalous émigrés d'Espagne avant l'édit de 1609 furent nombreux, nous pouvons citer, à titre d'exemple, 'Ahmad 'Al 'Garnāți qui enseigna à la medesrsa 'al Ma ${ }^{c}$ rid (27). 'Abū 'al Barakāt B Muk . ammad Ibn 'Usfūr qui donna des cours à la medersa Ibn țafargine et Abū 'al 'Abbās B Usfurī al Andalusī, qui enseigna a la medesra al usfuriyya (28).

Naturellement, comme toutes les autres medersas, al andalusiyya disposait d'un certain nombre de wakfs qui servaient à remunérer les professeurs et entretenir les locaux (29).

(17) Epalza, M, Petit, R, op. cit., p. 45.

(18) Zbiss, Gafsi, Boughanmi, Epalza, op. cit., 25

(19) Maamouri, T, ğamịa az zaytūna, Tunis, Maison arabe du livre, 1980, 77

(20) Cherif, $M H$, op. cit., 111

(21) Epalza, M, Petit, R, op. cit., 183.

(22) Zbiss, SM, monuments de Tunis, 55. Ben Mami, B op. cit., 363. Latham; J D, op. cit̂., 228.

(23) Ibn 'abĩ diāf, ithăâf, vol 2,35 .

(24) Ben Mami, B, op. cit., 364

(25) Ídem

(26) Ídem

(27) Az zarkašī, (mort 1488) Tārịn ad Dawlatayn, Tunis, Aka, 1966. 51.

(28) Epalza, M, Petit, R, op. cit., 300.

(29) Ces wakfs sont estimés à 9 boutiques et un hammân (Ben Mami, B, op. cit., 365) cet auteur n'indique pas la date. En $1256 / 1840$ ces wakfs comprennent 31 boutiques en plein medina de Tunis et 2 maisons (BNT, registre 21816,201 ) 


\section{Etude architecturale}

La medersa des andalous présente une disposition architecturale trés analogue aux autres medersas, mais cet un édifice simple, dépourvu de toute ornementation particuliere et surtout d'un portique bordant la cour à ciel ouvert ou Šahn. D'ailleurs I'état actuel de délabrement ne nous permet pas de suivre minitieusement cette architecture.

La façade donnant sur la rue Rakkāh est percée de trois fenêtres barraudées et d'une porte monumentale. (Fig. 3 ).

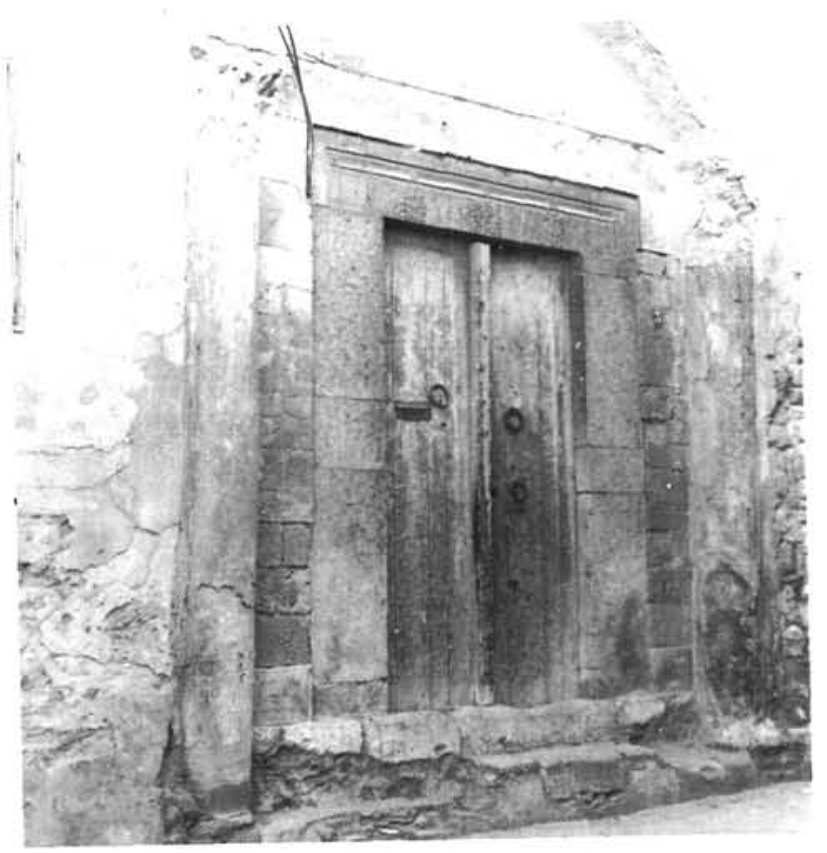

Fig. 3. Entrée de la Médersa des Andalous.

Au milieu de cette façade s'ouvre la porte d'entrée, à deux vantaux munis de trois heurtoirs (Midakkāt) à anneaux de fer, s'encadrant dans une jolie baie en fer à cheval à claveaux de grés coquillier (H̦arš) soutenue par deux colonnes à chapiteaux corinthiens. Cette porte à linteau droit est entourée d'un double encadrement; le premier est en pierre calcaire (Kadd dâl) et le deuxième en grés coquillier (Harš) garni d'un décor floral (rosettes et rinceaux) assez développé. 
Le seuil franchi, on se trouve dans un vestibule (sakīfa) bordé à sa droite de la salle d'ablutions (mĩda) et de salles d'aisance.

Ce vestibule donne accés à un patio (Șahnn) démuni de portique et de forme losangée $(11,51 \mathrm{~m}, 9,20 \mathrm{~m}, 9,53 \mathrm{~m}, 9,10 \mathrm{~m})$. Cette cour à ciel ouvert est dallée de kaḍdāl; sous ce dallage se trouve une citerne couverte (māgil) alors qu'un rebord de tuiles arrondies de couleur verte couronne la terrasse.

Autour de cette cour se disposent neuf chambres éclairées par des fenêtres donnant sur la cour (Fig. 4) et une mosquée (masğid); cinq chambres sont couvertes en berceau tandis que les quatre autres sont couvertes de charpente en bois.

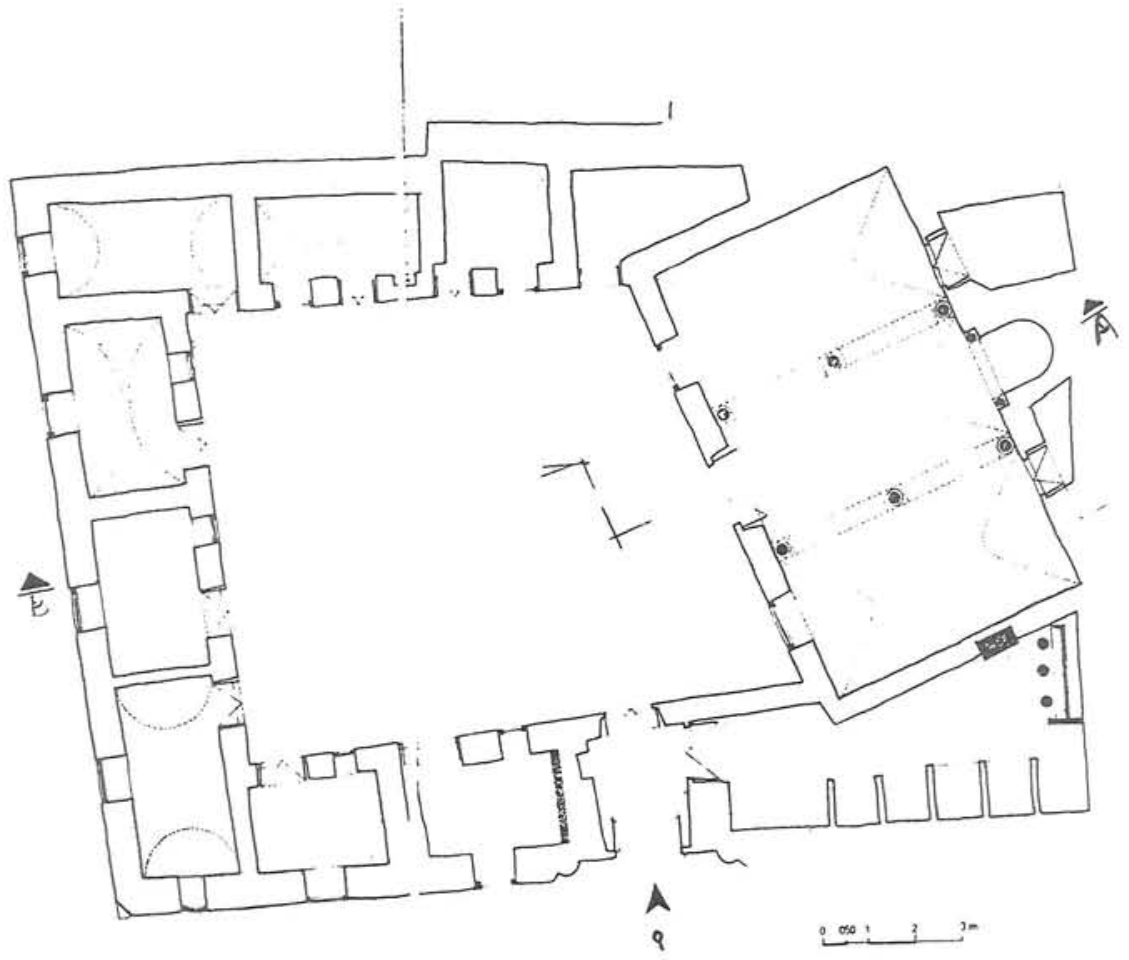

Fig. 4. Cour de la Médersa des Andalous

a. Plan géneral de la Medersa.

b. Coupes.

c, d, e, f. Portes et fenêtres sur la cour. 
LA MEDERSA DES MORISCOS ANDALOUS A TUNIS / EI Gaf́si
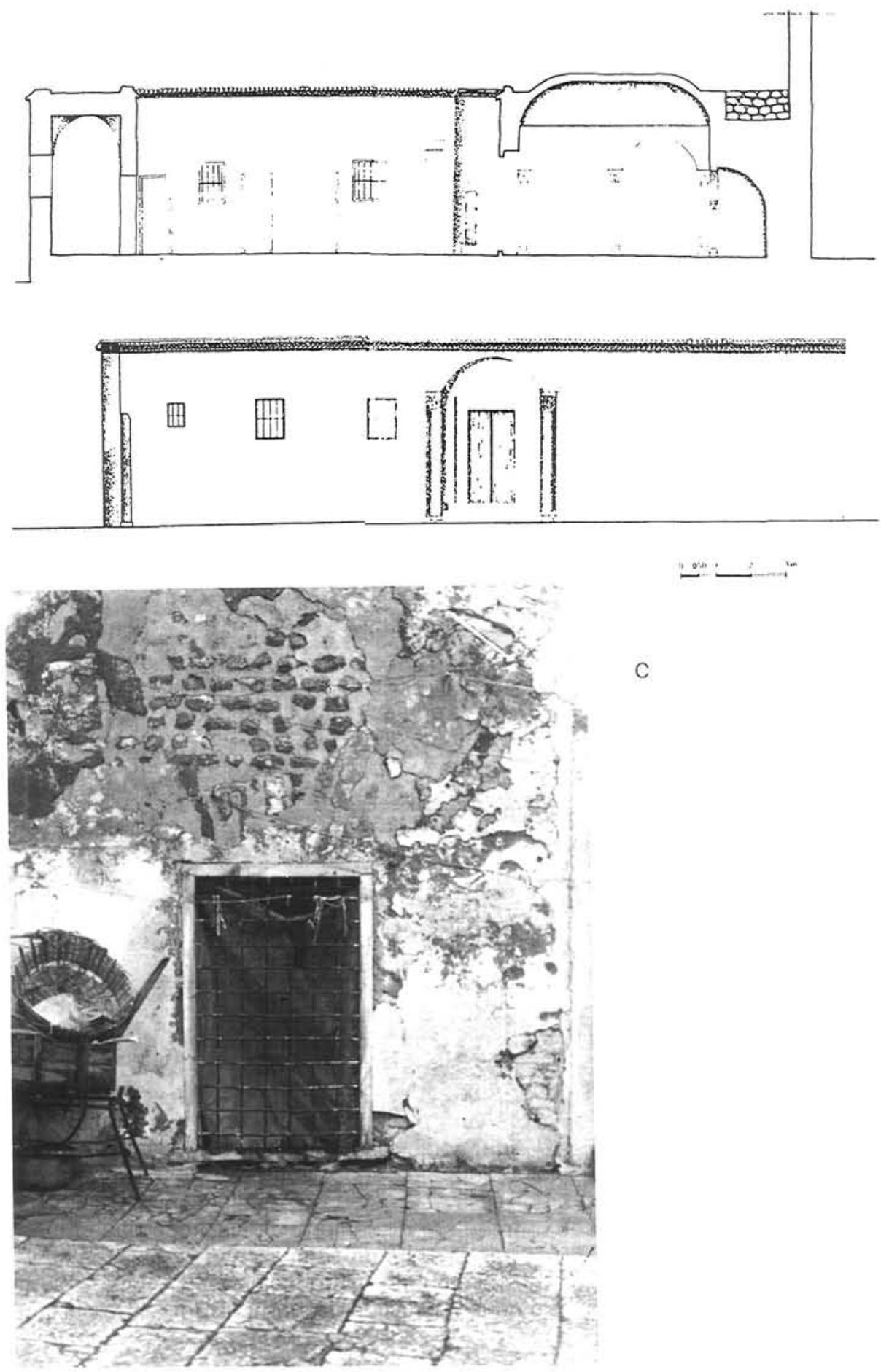

C 


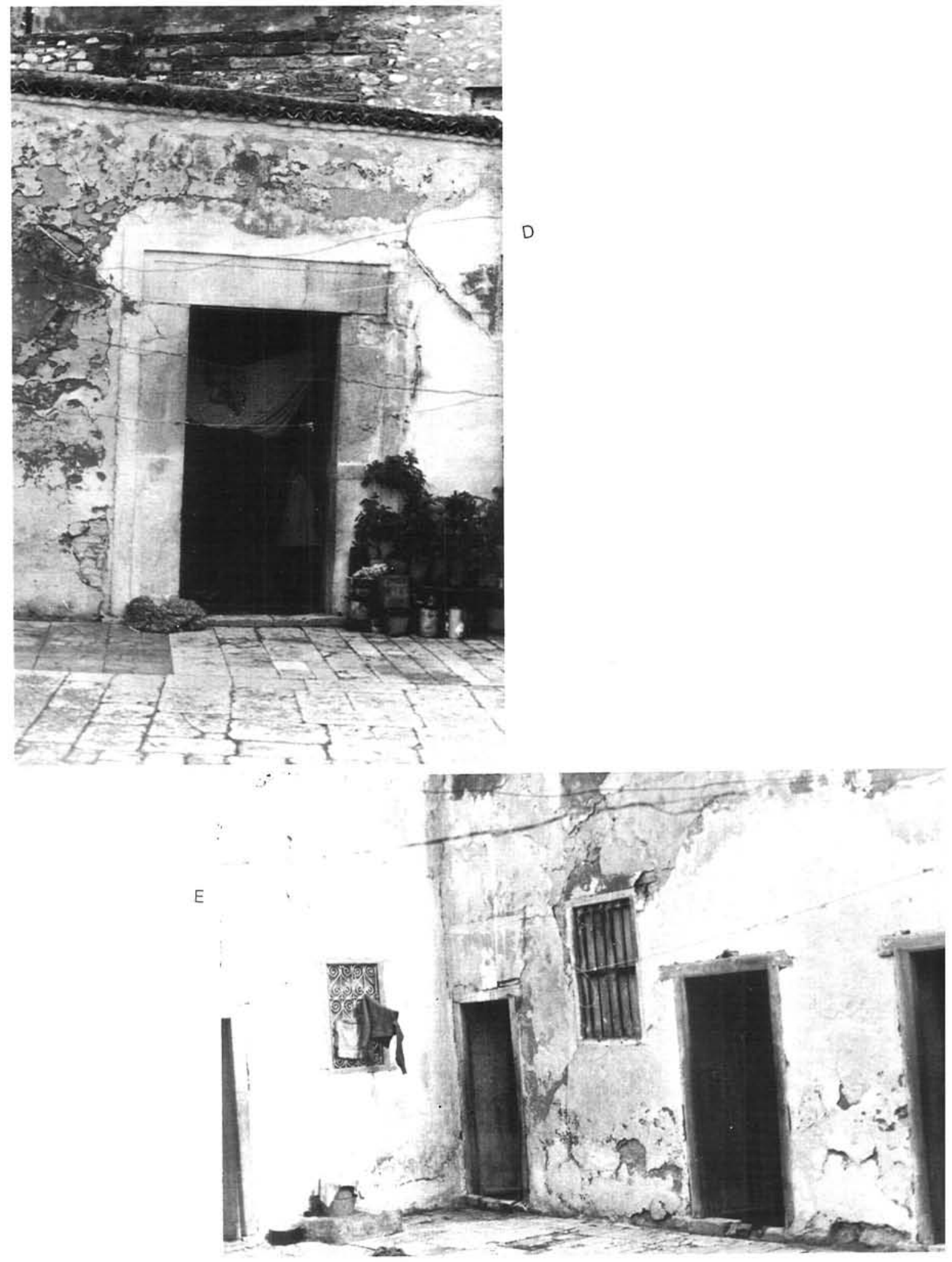




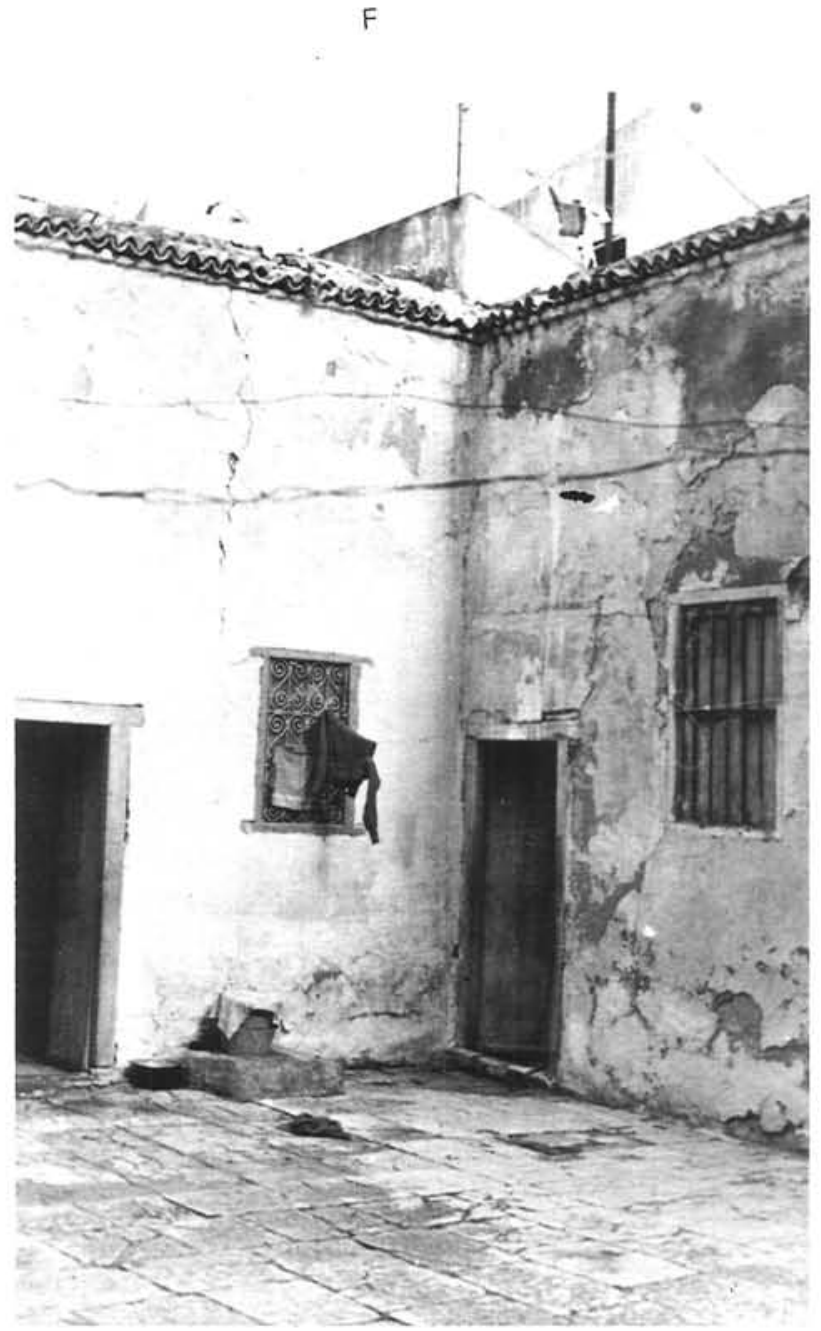


La porte de la mosquée est flanquée de deux fenêtres barraudées; elle comporte un linteau droit et deux montants en kaḍaal et donne accés directement à la salle de priere (Bayt aș șalāt). Celle-ci compte trois nefs et deux travées couvertes en berceau; la nef médiane repose sur deux rangées de colonnes à chapiteaux de style hafside (Fig. 5), trois de chaque coté. Au bout de cette nef et au milieu du mur de la kibla se creuse le mihrāb; il est entouré de 25 claveaux alternativement noirs et blancs reposant sur deux colonnettes à chapiteaux de style ottoman (volutes). Ce mihraāb s'inscrit dans un rectangle apparent avec boucle au sommet. A sa droite se trouve une niche aveugle (țāka) qui sert à déposer les livres; à sa gauche, le mur est percée d'une porte donnant accés à une chambrette qui pourrait servir au cheikh de la medersa.

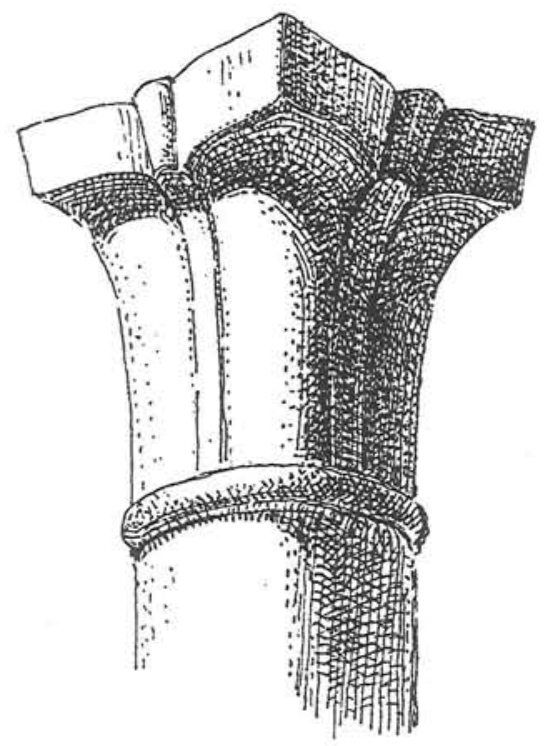

Fig. 5. Chapiteau tunisien d'époque hafside, selon G. Marçais.

Pour ce qui est des materiaux employés, nous observons de la brique pleine et des moellons.

\section{Conclusion:}

II importe pour conclure, de rappeler que la medersa des andalous à Tunis, ne diffère pas des autres, sur le plan fonctionnel et architectural, et reste jusqu'à present, malgré son affectation en oukala ou habitation collective, parmi les monuments andalous les plus significatifs. Cette medersa explique en partie quelques activités de cette colonie qui demeure tres importante dans la memoire collective de la population tunisienne. 